\title{
Diversity Performance of Off-body MB-OFDM UWB-MIMO
}

\author{
Marina Marinova, Arno Thielens, Emmeric Tanghe, Luigi Vallozzi, Günter Vermeeren, Wout Joseph, Senior \\ Member, IEEE, Hendrik Rogier, Senior Member, IEEE, Luc Martens, Member, IEEE
}

\begin{abstract}
This paper introduces a novel formalism to improve the performance of an off-body system by deploying multiple Ultra Wide Band (UWB) antennas, positioned strategically on the body. A methodology is presented for determining the optimal positions of UWB antennas on the body, necessary to provide a reliable Mult-Band Orthogonal Frequency Division Multiplexing (MB-OFDM) UWB diversity antenna system operating in the Federal Communications Commission frequency band between 3.1 and 10.6 GHz. By evaluating the diversity metric, using simulation and measurement data, it is shown that the performance of such a system is stable throughout the entire investigated frequency band for both indoor and outdoor environments. There is a good agreement between the simulated and measured diversity values with a deviation of less than $9 \%$.

Therefore, the proposed technique optimizes the antennas' positions for maximum diversity performance within a very broad frequency band, independent of the used wireless communication standard. Thus, the obtained diversity system might be used in any kind of wireless communication link within that frequency band, e.g. UWB-OFDM, UWB MB-OFDM, UWB or even narrowband transmission.
\end{abstract}

\section{INTRODUCTION}

In recent years, Body Area Networks (BANs) have been widely researched for a variety of potential applications, such as remote medical and healthcare services, surveillance/space applications, personal and business multimedia entertainment, sports training, and data transfer. BANs can be subdivided into three types, being in-body, on-body, and off-body systems. This characterization is based on the position of the nodes in these systems and on the intended communication link [1].

Due to the proximity of the human body, these types of wireless networks are subject to limitations in terms of radiated and consumed power, device size, and interference with coexisting wireless networks. Furthermore, in proximity of or inside the human body, the performance of the system does not only depend on the surrounding environment but also on the body shape and activities, the position of the antennas and their

This work was supported by the project IUAP- BESTCOM, "BElgian network on STochastic modelling, analysis, design and optimization of COMmunication systems" and by Research Foundation-Flanders (FWO-V); grant number: 3G004612.

Marina Marinova, Arno Thielens, Emmeric Tanghe, Günter Vermeeren, Wout Joseph, and Luc Martens are with Ghent University/iMinds, Dept. of Information Technology, Gaston Crommenlaan 8, B-9050 Ghent, Belgium, Fax: +32 933 14899, E-mail: marina.marinova@intec.UGent.be.

Marina Marinova, Luigi Vallozzi, and Hendrik Rogier are with Ghent University, Dept. of Information Technology, SintPietersnieuwstraat 41, 9000 Ghent, Belgium, Fax: +32 926 49969, E-mail:marina.marinova@intec.Ugent.be

Emmeric Tanghe and Luigi Vallozzi are Post-Doctoral Fellows of the FWOV (Research Foundation-Flanders) interaction with the body [1-4].

Moreover, it is important that BANs limit their radiated power in order to comply with exposure norms and keep the power consumption low as to guarantee long battery life. An interesting approach to meet these requirements is to make use of Ultra Wide Band (UWB) systems, which can provide very high data rate, low cost and low power consumption and emission [5], [6]. Additionally, the use of multiband OFDM helps to reduce the overall design complexity, improves the spectral flexibility, and helps to efficiently capture the multipath energy [7]. The reliability of the system can be further improved (thereby further increasing data rates, covered distances and spectral efficiency) by deploying multiple antennas positioned at strategic places on the body and by applying different diversity techniques for combining the signals received by those antennas [6], [7]. The antenna diversity overcomes performance degradation due to multipath fading of the signal, improves robustness of the system, increases the data rate, and, by using the proper space-time-frequency (STF) coding and modulation technique, increases the spectral efficiency [7]. Up to now, there are a large number of studies that have investigated on-body communication systems. Different channel models for systems operating at the $2.45 \mathrm{GHz}$ industrial, scientific and medical (ISM) band are presented in [8-12]. These channel models are based on measurement campaigns in various environments, including the anechoic chamber. Different channel characteristics, such as mean channel gain, shadowing and fast fading conditions are investigated in [9], while delay spread and path loss are studied in [10]. In addition, [11], [12] focus on Doppler spectrum and correlation. Furthermore, the on-body radio propagation is statistically analysed and evaluated in [13]. Less research results are found for higher frequency bands. In [4], a statistical model for the $4.5 \mathrm{GHz}$ narrowband channel is provided. [14] proposes a dynamic channel model for multi-sensor BANs for UWB (3-10 GHz). An analytical space-time channel model for UWB multisensor MIMO (multiple-input, multiple-output) BANs, operating in the 3-7 GHz band, is proposed in [15]. [16] investigates on-body channel models and system performance of an UWB cooperative BAN (CoBAN) with $5 \mathrm{GHz}$ bandwidth and proposes a method for calculating the diversity levels in a two-stage, single-hop CoBAN. The behavior of the frequencydomain and the delay-domain spatial correlation for systems operating at $3-6 \mathrm{GHz}$ and $3-10 \mathrm{GHz}$ is investigated in [17]. Different spatial diversity techniques for UWB on-body systems operating in the $3-10 \mathrm{GHz}$ band and using two receiving antennas are presented in [18], [19]. The frequency-space- 
polarization of an UWB MIMO system over three different frequency bands $(3-6,6-10,3-10 \mathrm{GHz})$ is investigated in [20]. In [21], measurement results are shown for an on-body UWB antenna diversity system.

In contrast to the on-body case, the studies on off-body communication systems are not that extensive and even fewer are those that investigate UWB systems or diversity systems. [22] considers a multiple antenna narrowband system operating at 5.8 and $10 \mathrm{GHz}$. A spatial diversity system operating at 5.8 $\mathrm{GHz}$ is represented in [23]. A few studies, such as [24], [25], investigate the propagation and the channel parameters for wideband and Ultra Wideband Systems.

The aim of this paper is to present a method for determining the minimum required number of UWB antennas for off-body MB-OFDM UWB MIMO communication and their optimal position on the body, necessary to provide a reliable diversity antenna system operating in the FCC (Federal Communications Commission) band between 3.1 and $10.6 \mathrm{GHz}$ [26]. Their optimal positions on the body are determined through simulations and validated by measurements. To the authors' knowledge, there are no comparable studies in the literature. This paper is organized as follows. Section II describes the UWB monopole antenna used for all simulations and measurements, and presents the simulation framework and the measurement setup. The simulation and measurement results are analyzed in Section III. Conclusions are drawn in Section IV.

\section{DesCription OF THE METHOdOLOGY}

\section{A. Objectives}

We consider an MB-OFDM UWB system that operates in the complete FCC frequency band between 3.1 and $10.6 \mathrm{GHz}$. The investigated system has $S$ subbands and $N$ subcarriers in each band, e.g. 14 subbands with 128 subcarriers each [7]. Even though an ultrawideband channel model [27] is available, due to the changing properties of human tissues with frequency, the system is tested for a set of three narrowband frequencies, being the two boundaries of the band (3.1 and $10.6 \mathrm{GHz}$ ) and the center frequency $6.85 \mathrm{GHz}$. Additionally we perform simulations and measurements for a set of another 7 frequencies, $[4,5,6,7,8,9,10] \mathrm{GHz}$, which we use for further validation of the method in Section III-B2. The system we consider is an MB-OFDM UWB system, which requires low interference between the separate bands [28]. For all cases, we focus on two environments: Urban Macrocell and Indoor Picocell, representing common real-life environments [29], [30]. The reliability of the system is tested by comparing simulation and measurement results for all combinations of frequencies and environments.

The method is based on simulation results obtained by the WINNER Phase II Channel Model version 1.1 [29] and the 3D finite difference time domain (FDTD) simulation platform SEMCAD X [31]. It is validated by actual measurements. The electromagnetic fields at $1 \mathrm{~cm}$ from the human body are calculated using the method presented in [30], [32]. To determine the positions of the nodes, we locate those areas of the body that provide low correlation and yield optimal diversity for the system. For the selected positions, the antenna diversity metric is calculated and compared with the theoretical values, in absence of the antennas.

\section{B. UWB Antenna}

The antenna used for simulations and measurements is a planar monopole antenna [33]. The antenna topology and its dimensions are presented in Fig. 1. The proposed antenna covers the frequency band $[2.9 ; 10.6] \mathrm{GHz}$. The antenna is implemented on a thin flexible polyimide foil with thickness of $25 \mu \mathrm{m}\left(\epsilon_{r}=3.2\right.$ at $\left.10 \mathrm{kHz}\right)$. The flexibility and the thin nature of the antenna makes it fully suitable for comfortable integration into garments as part of a wearable off-body wireless communication system [33]. More details about the antenna are given in [33].

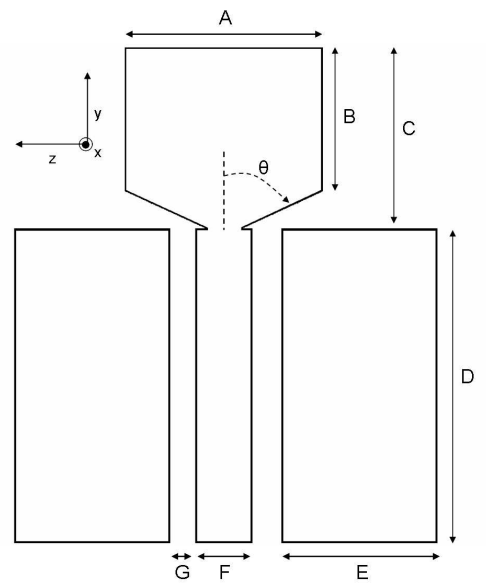

\begin{tabular}{|c|c|}
\hline \multicolumn{2}{|c|}{ Dimensions } \\
\hline$A$ & $17.85 \mathrm{~mm}$ \\
\hline$B$ & $12.72 \mathrm{~mm}$ \\
\hline$C$ & $16.05 \mathrm{~mm}$ \\
\hline$D$ & $27.89 \mathrm{~mm}$ \\
\hline$E$ & $14.04 \mathrm{~mm}$ \\
\hline$F$ & $5.06 \mathrm{~mm}$ \\
\hline$G$ & $0.16 \mathrm{~mm}$ \\
\hline$\theta$ & $68^{\circ}$ \\
\hline
\end{tabular}

Figure 1: Design and dimensions of the UWB Planar Monopole Antenna

\section{Simulation Framework}

In order to estimate the correct position of the nodes on the body (areas which provide low correlation), it is necessary to evaluate the electromagnetic fields around a human body exposed to a set of incoming plane waves. The initial frequency, chosen for determining the positions of the nodes, is $3.1 \mathrm{GHz}$ and we focus on the 'Indoor Picocell' environment. Subsequently, the system is verified at the other two chosen frequencies, being $6.85 \mathrm{GHz}$ and $10.6 \mathrm{GHz}$. In addition, the performance of the system is also tested for the second environment, 'Urban Macrocell', in combination with the three frequencies. The set of plane waves that create the electromagnetic field around the body is generated using the WINNER II Channel Model [29].

The different steps of the simulation framework are presented in Fig. 2.

1) Plane Wave Generation Using the WINNER II Channel Model (Step 1 in Fig. 2): Simulations are performed for all combinations of frequencies and environments. The environments used for the WINNER model are C2 Metropol and A1 In Building [29], which correspond to Urban Macrocell and Indoor Picocell, respectively. We simulate a set of channel 


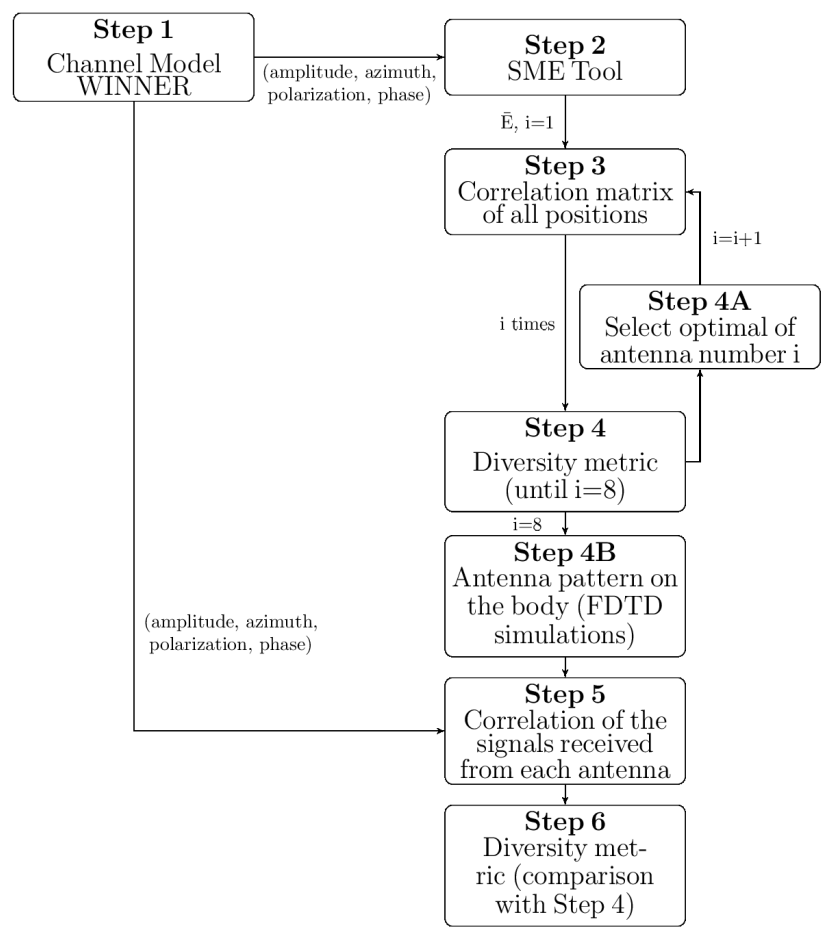

Figure 2: Flow graph of the simulation framework

snapshots for Non-Line-of-Sight (NLoS) conditions (Rayleigh fading channel). Although most diversity system parameters are typically defined for both NLoS and LoS propagation, MIMO systems provide better performance in NLoS propagation environment thanks to the multipath propagation and the restrictions for the power imbalance. Due to these reasons, we focus only on environments with NLoS propagation. However, beside the NLoS conditions, the off-body communication channel presents some specific challenges, such as body shadowing, resulting in effects such as power imbalance. For such propagation scenarios, the conventionally applied figures of merit only provide a partial view on the potential diversity gain.

Each sample includes values for amplitude, polarization, phase and azimuth angle for all incoming plane waves. As the publicly available codes for the WINNER model do not provide values for the elevation angles, we separately add such simulated values using [34]. The acquired data enable us to calculate the electromagnetic fields for each point around the body and to use the same set of plane waves for calculating the received signal at each antenna. The antenna characteristics we calculate from simulations are estimated assuming perfectly matched antennas.

2) Calculation of the electromagnetic fields around the body (Step 2 in Fig. 2): A set of plane waves produces an electromagnetic field around the body. These fields must be known in order to be able to estimate the best positions for the nodes on the body. Unfortunately, it is impossible to simulate those fields for every pair of azimuth and elevation angles of the incoming plane waves. Therefore, the ranges for these two angles have to be discretized in order to perform simulations for only a limited number of basic incident plane waves. The fields around the body, induced by this set of incident plane waves, are called basic field distributions (BFDs). These BFDs can be used for estimating the fields induced by waves impinging from any direction.

Every plane wave is characterized by its amplitude, phase, polarization and incoming azimuth and elevation angles $(\phi, \theta)$. It is estimated using the extracted BFDs. The method is explained in detail in [30], [34], [35]. For the discretization of the azimuth angle, we use $\Delta \phi=20^{\circ}$. For the elevation angle, we use $\Delta \theta=4^{\circ}[30]$.

To calculate the electromagnetic fields at a $l \mathrm{~cm}$ distance from the body we rely on the Statistical Multi-path Exposure (SME) method developed in [30], [34], [35]. The simulations yield the electromagnetic field at about 63,000 different points. This number can be reduced in a number of ways.

As the system should be robust, harmless to the wearer, implementable in wearable garments and applicable for different scenarios, certain body parts are not considered to be suitable for deploying an antenna. Therefore, the lower part of the body, together with the arms and the sides of the torso are excluded from the list of suitable positions. The head is also excluded as antenna position, since deploying an antenna on the head is impractical and unaesthetic in most applications. Therefore, only positions on the shoulders as well as on the front and the back of the torso are considered to be suitable. However, the excluded areas can be useful in specific applications, such as for safety forces. Also for these scenarios, our technique can be optimized and used to include these areas.

Furthermore, as the antennas have actual physical dimensions, we must also ensure that they cannot overlap. Therefore, the body is subdivided in squares with dimensions $25 \times 25 \mathrm{~mm}$. This is done by representing positions on the body in polar coordinates and by sampling in $z$ and in $\varphi$, with $\Delta z=25$ $\mathrm{mm}$ and $\Delta \varphi=10^{\circ}$. The only field positions remaining after introducing this sampling scheme are the ones at the crossings of the $z$ and $\varphi$ lines, which results in 794 points. For these positions, the correlation matrix is calculated and further used for determining the optimal locations to deploy antennas on the body.

3) Correlation (Step 3 in Fig. 2): A requirement to obtain good performance in a mobile diversity system is that the correlation coefficient remains below 0.5 [36]. The correlation is given by [37]:

$$
\rho_{X, Y}=\frac{\operatorname{cov}(X, Y)}{\sigma_{X} \sigma_{Y}},
$$

where $\operatorname{cov}(\cdot)$ is the covariance and $\sigma$ is the variance.

Correlation analysis in on-body [12] and off-body [38] radio channels resulted in a minimum achieved correlation coefficient of $\rho=0.19$ for antennas positioned on the head and the waist. Therefore, we design a system that realizes $\rho<0.19$, for antennas positioned only on the front and back of the torso.

4) Diversity Metric (Step 4 in Fig. 2): Diversity metric $D(R)$ is used for quantifying the available diversity order for a given Rayleigh fading MIMO system with correlation matrix 
$R$. It is given by [39]:

$$
D(R)=\left(\frac{\operatorname{tr}(R)}{\|R\|_{F}}\right)^{2},
$$

where $\operatorname{tr}(\cdot)$ denotes the matrix trace, and $\|\cdot\|_{F}$ denotes the Frobenius matrix norm. The metric varies between $l$ and $L$, where $L$ is the number of antennas used for achieving diversity. If $D=1$, then there is no diversity gain and if $D=L$ we obtain maximum diversity in the channel [39]. This metric allows us to compare different diversity systems based on their performance in a NLoS environment.

The diversity metric is chosen as a criterion for determining the optimal positions of the nodes on the body. The first two nodes are placed at the positions with the lowest mutual correlation. Each additional antenna is chosen as the one that minimizes the Frobenius norm of the correlation matrix $(\operatorname{tr}(R)=L)$ (Step 4A in Fig. 2).

The maximum number of antennas considered for further investigation is 8 . For all simulations and calculations, we neglect mutual coupling between the antennas. This approximation will be verified in Section III-B1.

For the already identified positions, an FDTD simulation is performed with an antenna placed on a heterogeneous realistic human body phantom (Virtual Family Male - Duke [40]) (Step 4B in Fig. 2). As a result, the antenna characteristics (radiation pattern, gain, reflection coefficient), are extracted and the correlation between the antennas (Step 5 in Fig. 2) in the system and the diversity metric (Step 6 in Fig. 2) are calculated.

As decided in Section II-C3, we design a system with correlation of $\rho \lesssim 0.19$. This corresponds to threshold values for the diversity metric of $1 \leq D \leq 6.39$ for $1 \leq L \leq 8$. For $L=5$ antennas, the diversity metric now equals $D=4.37$.

\section{Measurement Setup}

To verify our new formalism to determine the ideal antenna positions, we perform measurements in an indoor environment with NLoS conditions and in an anechoic chamber. As a transmitter (Tx), we use a UWB horn antenna (operating between 1.12 and $12.4 \mathrm{GHz}$ with a maximum gain of $12.55 \mathrm{dBi}$ ). The gain of the horn antenna for all measured frequencies is given in Table I. The dimensions of the Tx are width $A=245 \mathrm{~mm}$ and height $B=138 \mathrm{~mm}$ which corresponds to a maximum overall dimension $D=281 \mathrm{~mm}$. With these parameters, the distance between the $\mathrm{Tx}$ and receiver ( $\mathrm{Rx})$ should be at least $5.59 \mathrm{~m}$ (in the case of $10.6 \mathrm{GHz}$ ), to obtain far field propagation. In the measurement setup, the minimum distance between the $\mathrm{Tx}$ and $\mathrm{Rx}$ is the one of the shortest specular reflection paths, which is approximately 15 $\mathrm{m}$ (the direct path between the $\mathrm{Rx}$ and $\mathrm{Tx}$ is $4 \mathrm{~m}$ but due to the absorbing wall situated between them and the horn antenna being rotated at $45^{\circ}$, the Rx cannot receive any LoS components). To record the data, we rely on a 4-port network analyzer (PNA-X Microwave Network Analyzer N5242A by Agilent Technologies [41]), which simultaneously measures the signal from the Tx (UWB horn antenna) and three on-body antennas. Fig. 3 presents the measurement configuration.

\begin{tabular}{|c||c|c|c|c|c|}
\hline \multicolumn{7}{|c|}{ Gain of the horn antenna } \\
\hline Freq., (GHz) & 3.1 & 4 & 5 & 6 & 6.85 \\
\hline Gain, (dBi) & 8.47 & 9.81 & 11.01 & 11.45 & 11.09 \\
\hline \multicolumn{7}{|c|}{} \\
\hline Freq., (GHz) & 7 & 8 & 9 & 10 & 10.6 \\
\hline Gain, (dBi) & 10.73 & 11.06 & 11.16 & 11.51 & 11.95 \\
\hline
\end{tabular}

Table I: Gain of the horn antenna for all measured frequencies

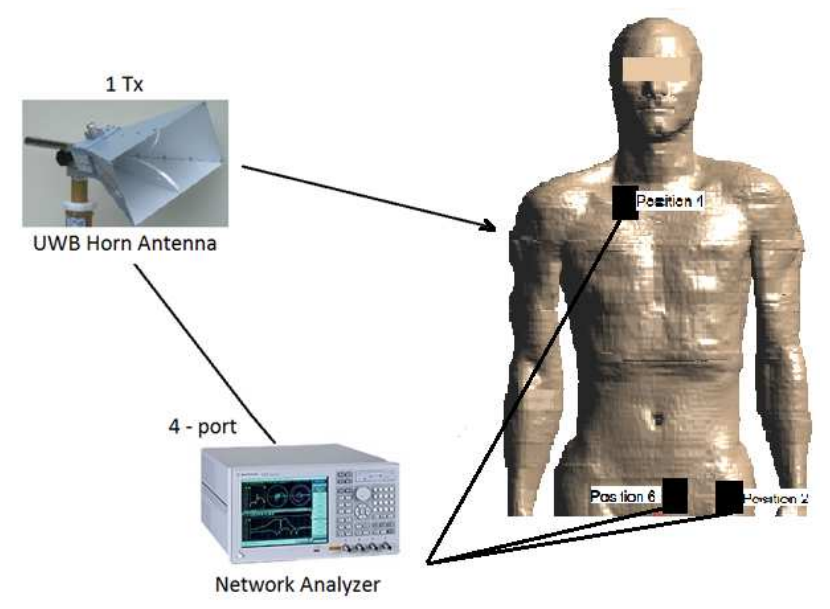

Figure 3: Measurement configuration

As it is not possible to measure all eight antennas simultaneously, we perform multiple measurements for different configurations of three antennas. The antennas are placed in a frame of expanded polystyrene with an approximate thickness of $1 \mathrm{~cm}$ and were attached to the clothes of a subject at the computed optimal positions (Fig. 4). The cables, connecting the antennas to the network analyzer, are decoupled using ferrite beads.

In order to reliably calculate the correlation between the

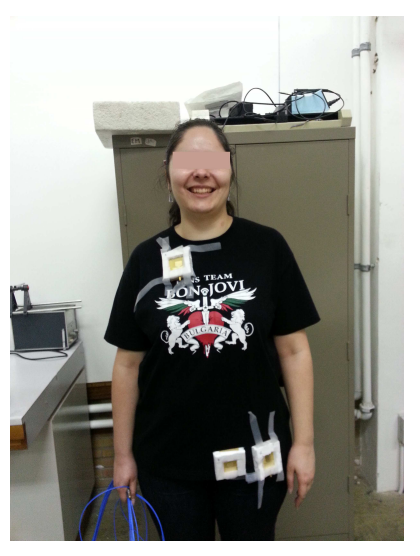

(a)

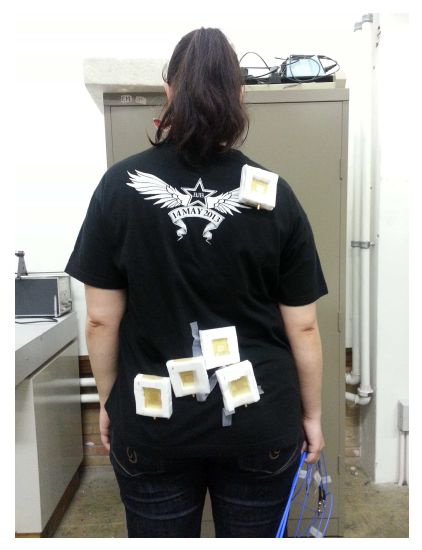

(b)
Figure 4: Antennas attached to the body: front (a) and back (b)

antennas, it is necessary to have a sufficiently large number of observations (channel snapshots). We measure 1000 channel snapshots by recording 1000 sweeps of the S-matrix at one frequency point in an indoor environment, while our test 
person is walking at a constant speed of approximately 1 $\mathrm{m} / \mathrm{s}$ along a predefined path. We performed measurements at $[3.1,4,5,6,6.85,7,8,9,10,10.6] \mathrm{GHz}$. With regard to the above mentioned parameters, the maximum Doppler shift is equal to $f_{d}=34.13 \mathrm{~Hz}$ at $10.6 \mathrm{GHz}$, which corresponds to a coherence time $T_{c}=29 \mathrm{~ms}$. The spatial sampling time $T_{s}$ is the time interval between recording two channel snapshots and $T_{s}>T_{c}$ is required, in order to ensure that the channel changes substantially between the recorded channel snapshots. Considering the settings of the network analyzer, we obtain $T_{s}=60 \mathrm{~ms}$. Therefore, we can conclude that the channel changes substantially between each snapshot. $T_{\text {snapshot }}$ is the time required for measuring the complete channel $\mathrm{S}$ matrix of one sample. This value must be smaller than the coherence time in order to ensure the static nature of the channel during each snapshot measurement. $T_{\text {snapshot }}<20$ $\mathrm{ms}$ and, therefore, the channel can be considered static during the measurement of each snapshot. Using the measurement data, we calculate the total received power of each antenna, its reflection coefficient $S_{11}$, and the mutual coupling between the antennas.

Additionally, for comparison, we also measure the "most commonly used positions" (Fig. 6) of the on-body antennas. For the measurements of the antennas situated on the arms, the hands of our test subject remain static during her walk.

A scheme and photograph of the measurement environment and the measurement path are shown in Fig. 5. All cupboards and electricity boards have metallic surfaces. The walls and ceiling of the measurement lab are made of concrete. Part of the walls are windows with wooden frames. The ceiling is crossed by metallic pipes and the floor is covered by linoleum.

\section{Simulation And Measurement Results AND DISCUSSIONS}

In the following section, the simulation and measurement results for the proposed system are presented. Section III-A explains the selection of the optimal antenna positions and how the simulated performance of such a system compares to the one of a system using the most commonly used positions to place on-body antennas. These positions, shown on Fig. 6, were obtained from [2], [9], [12]. They are denoted as the most common positions from now on. Further on, the measurement results and their comparison with those obtained through simulation are presented in Section III-B.

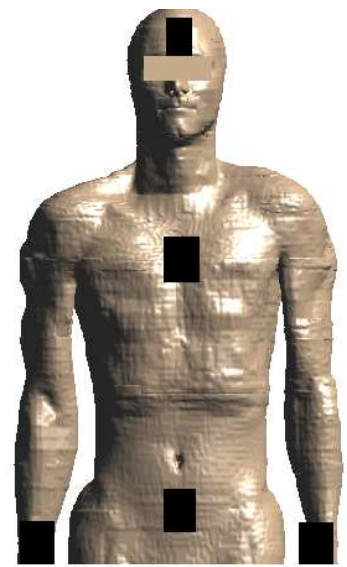

(a)

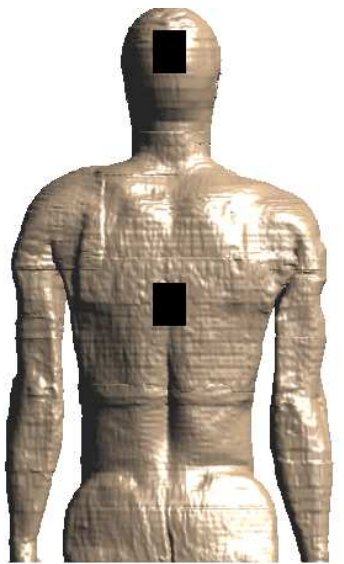

(b)
Figure 6: Most common antenna positions on the front (a) and back (b) of the human model (introduced by black boxes)

\section{A. Diversity metric}

Using the WINNER II channel model, a set of 5000 channel snapshots is simulated. For every set of snapshots, the SME tool ([30], [32], [34], [35]) evaluates the electric fields. The correlation between the received electric fields at these points is calculated. All simulations are performed considering vertical polarization and neglecting the mutual coupling between the antennas on the body. In addition,

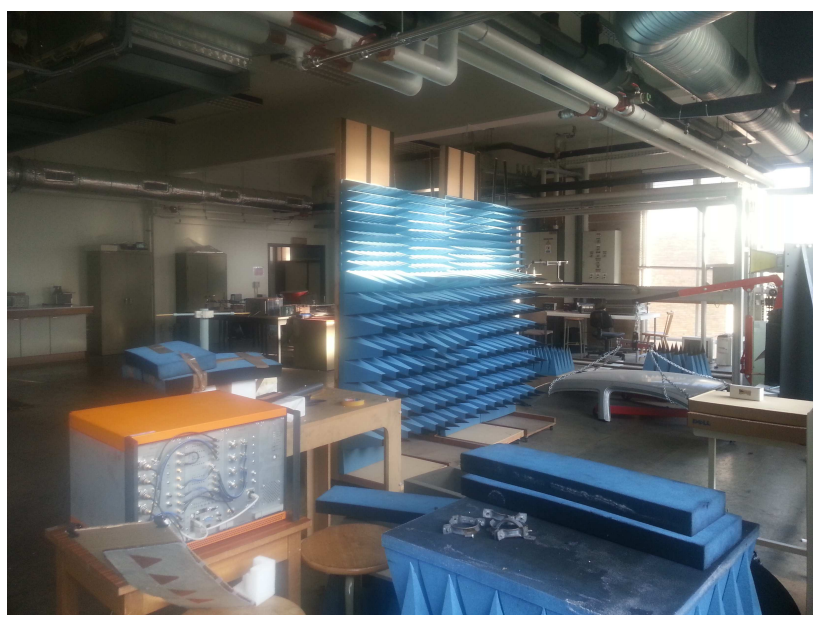

(b)

Figure 5: Scheme and photograph of the measurement environment and visualization of the measurement path indicated with a dotted line 
we assume that if a combination of $n$ antenna positions is optimal (providing maximally $D_{n \text { antennas }}(R)$ ), we can reuse that same combination and calculate the next position to obtain an optimal configuration of $n+1$ antennas (the value of $D_{n+1}$ antennas $(R)$ is the maximum achievable one).

1) Selection Based on Received Electric Fields: Initial simulations and calculations are performed at $f=3.1 \mathrm{GHz}$, for a A1 In Building environment (Section II-C). Using the calculated correlation matrix for the potential positions and the diversity metric (2), the positions that provide low correlation and maximum $D(R)$ are chosen. $D(R)$ is also calculated 1000 times for a random choice of antennas. Fig. 7 compares the results for the simulated set of data with those for a random choice of position (calculated median of all random realizations). The proposed method provides better results than randomly positioning the nodes on the body, even when selecting the best random realization. As explained in Section II-C4, the maximum $D(R)=n$, where $n$ is the number of antennas. For example, for $n=5, \max (D(R))=5$, $D_{\text {optimal positions }}=4.99, D_{\text {random positions-median value }}=$ 4.17. This result is to be expected considering the fact that the randomly generated values were simulated using the function rand in MATLAB and this function provides pseudorandom uniformly distributed values.

As we can see from Fig. 7, when the system consists of 1 or 2 antennas, the value of the diversity metric is close or equal to $\max (D(R))$. Therefore, in the sequel we will show only the values for systems consisting of 3-8 antennas. In addition, Fig. 7 also plots the threshold values for the diversity metric $D_{\rho=0.19}$. The proposed system has better performance for a system with 2-8 antennas and $D_{\text {optimal positions }}$ is larger than $D_{\rho=0.19}$ with a difference between $3.5-19.7 \%$, depending on the number of antennas. For $n=5, D_{\text {optimal positions }}$ is $12.5 \%$ higher than $D_{\rho=0.19}$.

The obtained eight optimal positions with maximum diversity are shown in Fig. 8. The first two nodes are logically positioned on the front and the back of the human body model, although they are usually situated in the center of the chest and the corresponding position in the back [2], [9], [12]. The rest of the positions are different from those typically applied in literature [2], [9], [12]. We should also take into account the fact that only three of the commonly used positions (Fig. 6) are situated on the torso (waist front, chest front and back), which is the area chosen for placing our antennas.

2) Selection including the UWB Antenna: For each antenna position, an FDTD simulation of the antenna with a heterogeneous realistic human body phantom at $3.1 \mathrm{GHz}$ is performed and the radiation patterns, gains, reflection coefficients, etc. of the antennas are extracted. Using these data and the 5000 channel snapshots, we calculate the total values of the received power for each antenna and construct a new correlation matrix, which now includes the influence of the antennas (Step 5 in Fig. 2). We then calculate the diversity metric and compare these results with the initial ones, calculated without antennas. Furthermore, we compare the performance of this system with two other sets of antennas. The first one is a system with randomly positioned antennas. Amongst the 1000 sets of

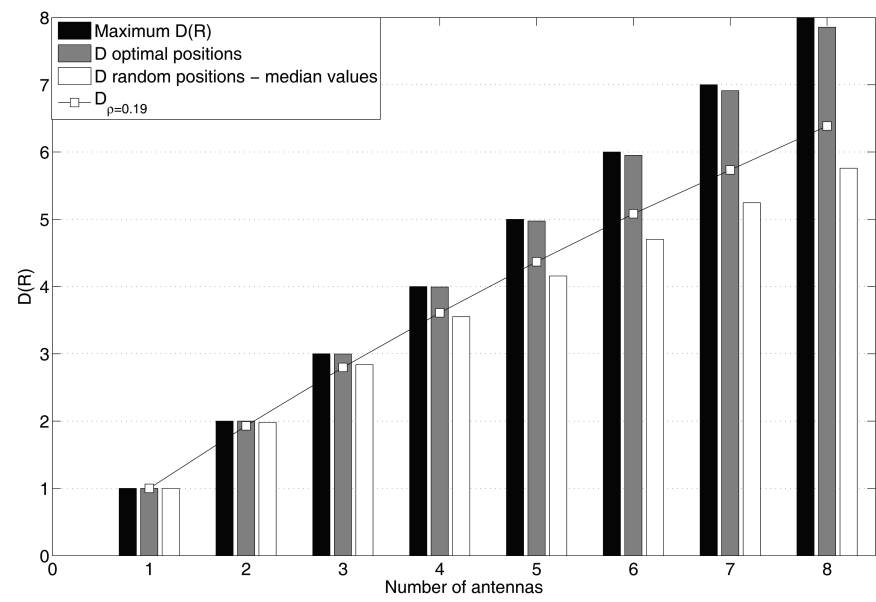

Figure 7: Comparison of the diversity metric obtained for: a) Maximum $D(R)$ - maximum possible diversity in the channel; b) Doptimal positions - values calculated using the optimal antenna positions obtained through the proposed method; c)

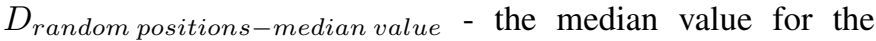
1000 sets of randomly chosen antennas; d) $D_{\rho=0.19}$ - the values for the diversity metric when the branches are equally correlated and $\rho=0.19$

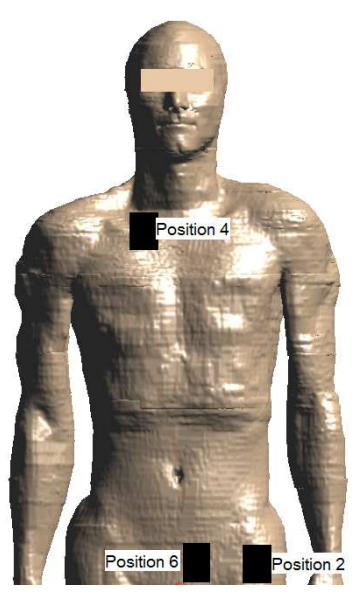

(a)

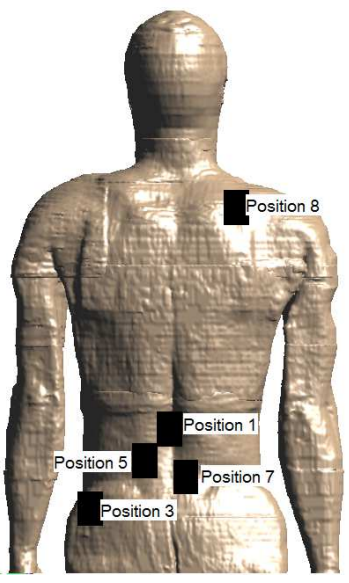

(b)
Figure 8: Optimal positions on the front (a) and back of the human model (b)

eight antenna positions we take the ones that provide the best performance. The second set of antenna positions are the most commonly used positions [2], [9], [12]. For all positions from these two sets, we apply the same procedure as for the optimal antenna configuration. The outcome of these comparisons is presented in Fig. 9. The performance of our proposed system is better than the randomly positioned antennas (Fig. 9). On average the values of $\mathrm{D}$ for the proposed system are $16.1 \%$ higher than the randomly positioned ones (this number varies between 7.5 and $24.2 \%$, depending on the number of antennas), i.e. for $n=5 D(R)_{\text {optimal positions-with antenna }}=4.996$ and $D(R)_{\text {best realization-random with antenna }}=4.22$ which results in $\Delta D=15.5 \%$.

From Fig. 9 we also see that a system with the most 


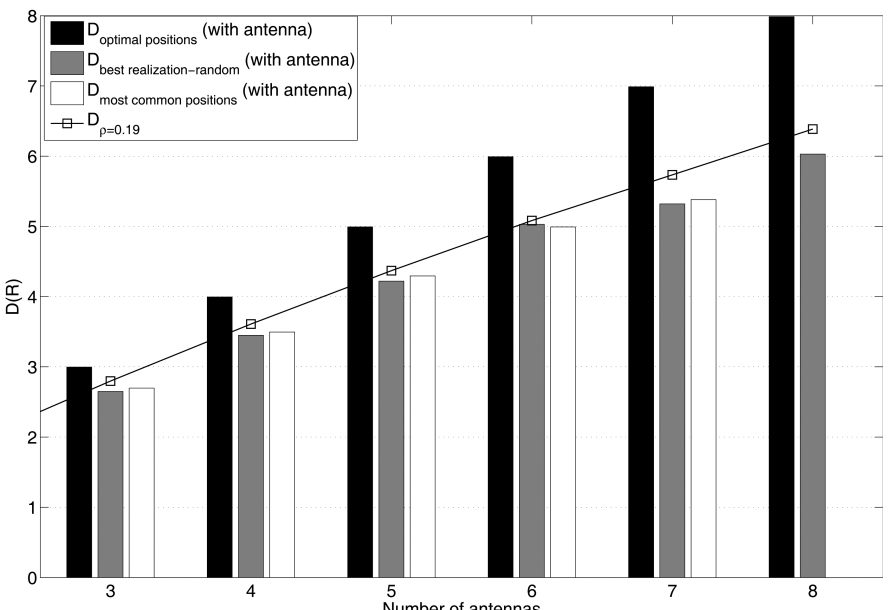

Figure 9: Comparison of the diversity metric values for a system consisting of realistic UWB antennas placed at: a) optimal antenna positions; b) Best realization of random choice of antennas; c) most commonly used positions; d) $D_{\rho=0.19}$ - the values for the diversity metric when the branches are equally correlated and $\rho=0.19$

commonly used positions exhibits worse performance than the one we constructed. On average, the proposed method provides approximately $13.4 \%$ higher values for $\mathrm{D}$ than the most commonly used positions (this value varies between 4.6 and $22.6 \%$ depending on the number of antennas), i.e.

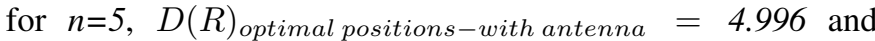
$D(R)_{\text {most common positions }- \text { with }_{a} \text { ntenna }}=4.29$, thereby indicating the necessity of such a method.

In order to show that the system can operate throughout the investigated frequency band, we calculate the diversity metric for $6.85 \mathrm{GHz}$ and $10.6 \mathrm{GHz}$ and compare the values with those we obtained for $3.1 \mathrm{GHz}$. The result of this comparison is presented in Fig. 10. We notice that the values for the three frequencies are similar (less than $0.5 \%$ difference for both investigated environments). Therefore, we can conclude that the performance of the system is reliable in the frequency band $[3.1,10.6] \mathrm{GHz}$.

The diversity metric for the system is also calculated for the C2 Metropol environment and the results are compared with those for the A1 In Building. The antenna system provides nearly identical behavior for both environments (less than $1 \%$ difference for all frequencies). Therefore, we can assume that our system may be applied for both indoor and outdoor applications.

\section{B. Measurement Results}

1) UWB Antenna: In this section we investigate the influence of the positioning of the antenna on the body on its characteristics and the coupling between the antennas. In terms of reflection coefficient, it was found that the UWB antennas' $\left|S_{11}\right|$ remains below $-6.8 \mathrm{~dB}$ for all considered positions on the body. Moreover, for most positions, the return loss is significantly better than $10 \mathrm{~dB}$.

The mutual coupling between all combinations of antennas is

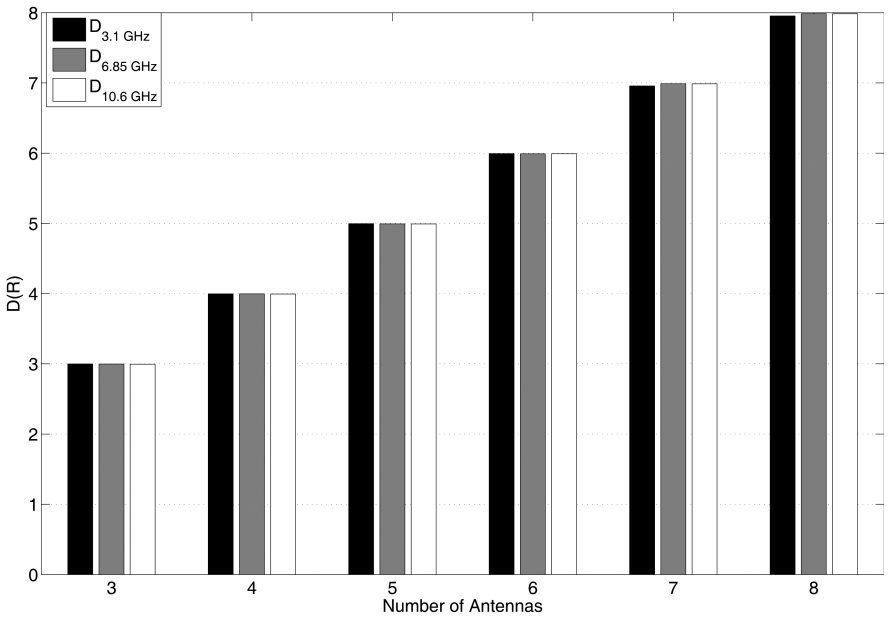

Figure 10: Diversity metric for the optimal antenna position for A1 In Building environment at the three investigated frequencies $[3.1 ; 6.85 ; 10.6] \mathrm{GHz}$

measured in an anechoic chamber. Depending on the position of the antennas on the body, the mutual coupling varies between -30 (mean value of the mutual coupling between antennas 2 and 7 ) and $-80 \mathrm{~dB}$ (mean value of the mutual coupling between antennas 3 and 7 ). From the measurement data it can also be concluded that at the higher frequencies the values of the mutual coupling are lower thanks to the better isolation between the antennas.

For all antenna pairs, the mutual coupling remains below -15 $\mathrm{dB}$ over the investigated frequency band and, as stated in [18], mutual coupling below that value can be neglected. Therefore, our assumption that the mutual coupling between the antennas can be neglected is valid.

2) Comparison Between Simulation and Measurement Results: Based on the measurement data, for each antenna we evaluate the received power for the set of three investigated frequencies in an indoor environment. Then, we calculate the diversity metric for the system and compare the results with those from the simulations. This enables us to compare between the system we developed and the one using the most commonly used positions.

Fig. 11 shows the values of the diversity metric calculated using the measurement and simulation data at $3.1 \mathrm{GHz}$. The simulated system has better performance than the measured one with a difference of less than $7.9 \%$. The optimal positions perform better than the $D_{\rho=0.19}$ value (proposed in Section II-C3), for both simulation and measurement results. Table II shows the values for the diversity metric for a system of 5 antennas. The comparison is made between the simulation and measurement results, for the optimal antenna positions, and the measurement results, for the most commonly used antenna positions, for the full set of measured frequencies, including the three investigated ones. The deviation is calculated with reference to the measurement value for the optimal antenna positions. The difference between the simulation and measurement results for the optimal antenna positions is less than $6.4 \%$. Therefore, we conclude that the results agree well. From the comparison between the measurement data for the 


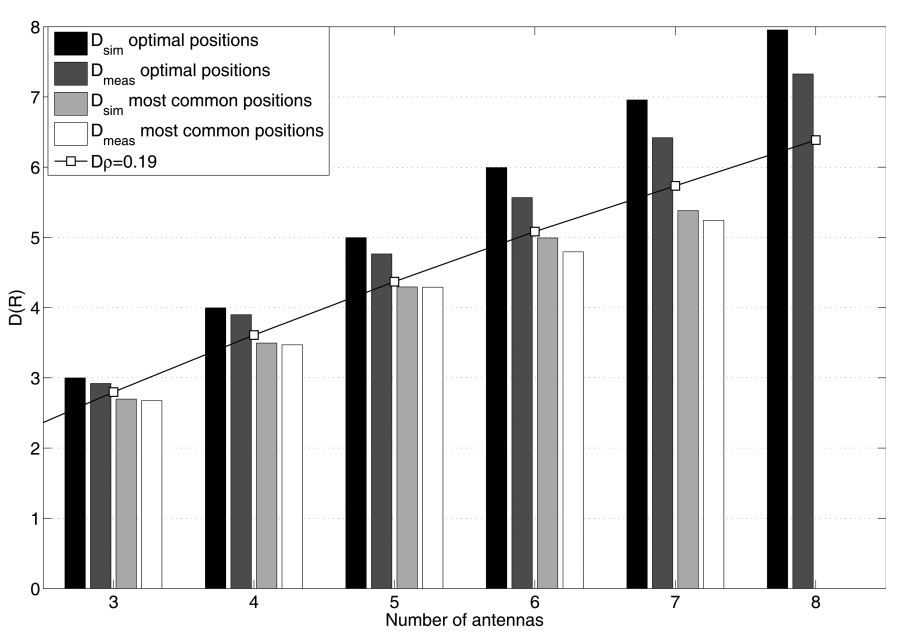

Figure 11: Diversity metric for the optimal antenna positions and the most common antenna positions: measurement versus simulation at $3.1 \mathrm{GHz}$

\begin{tabular}{|c||c||c|c||c|c|}
\hline \multicolumn{5}{|c|}{ Diversity metric D for NLoS A1 In Building environment } \\
\hline \multirow{2nnyyy}{*}{$\begin{array}{c}\text { Frequency, } \\
(\mathbf{G H z})\end{array}$} & $\begin{array}{c}\text { Reference } \\
\text { value } D_{\text {meas }}\end{array}$ & \multicolumn{2}{c|}{ Optimal positions } & \multicolumn{2}{c|}{ Most common positions } \\
\cline { 3 - 6 } & $D_{\text {simulation }}$ & $\Delta D \%$ & $D_{\text {meas }}$ & $\Delta D \%$ \\
\hline $3.10 \mathrm{GHz}$ & 4.76 & 5.00 & 4.9 & 4.29 & 10 \\
\hline $4.0 \mathrm{GHz}$ & 4.79 & 4.99 & 4.2 & 4.39 & 8.4 \\
\hline $5.0 \mathrm{GHz}$ & 4.72 & 4.98 & 5.5 & 4.51 & 4.5 \\
\hline $6.0 \mathrm{GHz}$ & 4.81 & 4.99 & 3.7 & 4.53 & 5.8 \\
\hline $6.85 \mathrm{GHz}$ & 4.69 & 4.99 & 6.4 & 4.59 & 2.2 \\
\hline $7.0 \mathrm{GHz}$ & 4.90 & 4.99 & 1.8 & 4.63 & 5.5 \\
\hline $8.0 \mathrm{GHz}$ & 4.94 & 4.98 & 0.8 & 4.66 & 5.7 \\
\hline $9.0 \mathrm{GHz}$ & 4.87 & 4.99 & 2.5 & 4.67 & 4.1 \\
\hline $10.0 \mathrm{GHz}$ & 4.97 & 4.99 & 0.4 & 4.52 & 9.1 \\
\hline $10.60 \mathrm{GHz}$ & 4.98 & 4.99 & 0.2 & 4.69 & 5.9 \\
\hline
\end{tabular}

Table II: Comparison between the simulation and measurement values in terms of diversity metric, at the three investigated frequencies for the optimal positions and deviation of the values for the optimal and most common positions with regards to $D_{\text {meas optimal positions, }}$, for a system of $n=5$ antennas

optimal antenna positions and the most commonly used ones, we notice that the largest deviation is obtained for $3.1 \mathrm{GHz}$ $(10 \%)$. For the rest of the frequencies, the deviation varies between $9.1 \%$ and $2.2 \%$ with mean value $6.1 \%$. From the data we can see that a system using the optimal antenna positions, provides very stable performance with deviation between the minimum and maximum value of $5.8 \%$. Compared to the most commonly used positions, without including positions on the head, the performance of the system constructed using the proposed method is at least $7 \%$ better, despite the fact that the antennas on the head are better isolated from those on the torso and arms. For $n=5$, the deviation of the diversity metric for the most commonly used positions with regards to the one for the optimal antenna positions is $13.5 \%$. This is also the case when we consider only the most common positions, which are situated on the torso (deviation of $D$ is $15.7 \%$ with respect to any system that uses three of the optimal antenna positions). The values for the three investigated frequencies are very similar (less than $8.2 \%$ deviation). They are shown in Table III. The highest values are achieved for $10.6 \mathrm{GHz}$. This is to be expected given the smaller wavelength, which results in a better isolation between the antennas. Hence, we conclude

\begin{tabular}{|c|c|c|c|c|c|}
\hline \multicolumn{6}{|c|}{ Diversity metric for NLoS A1 In Building Environment for the three investigated frequencies } \\
\hline \multirow{2}{*}{$\begin{array}{c}\begin{array}{c}\text { number of } \\
\text { antennas }\end{array} \\
\end{array}$} & \multirow{2}{*}{$\begin{array}{c}\text { Reference value } \\
D_{\text {meas }} 3.1 \mathrm{GHz}\end{array}$} & \multicolumn{2}{|c|}{$6.85 \mathbf{G H z}$} & \multicolumn{2}{|c|}{$10.6 \mathbf{G H z}$} \\
\hline & & $D_{\text {meas }}$ & $\Delta D \%$ & $D_{\text {meas }}$ & $\Delta D \%$ \\
\hline 2 antennas & 2.00 & 1.99 & 0.45 & 2.00 & 0.18 \\
\hline 3 antennas & 2.92 & 2.95 & 1.00 & 2.99 & 2.51 \\
\hline 4 antennas & 3.90 & 3.88 & 0.40 & 3.99 & 2.26 \\
\hline 5 antennas & 4.76 & 4.69 & 1.50 & 4.98 & 4.53 \\
\hline 6 antennas & 5.57 & 5.56 & 0.14 & 5.96 & 7.10 \\
\hline 7 antennas & 6.42 & 6.44 & 0.30 & 6.94 & 8.11 \\
\hline 8 antennas & 7.33 & 7.35 & 0.34 & 7.88 & 7.53 \\
\hline
\end{tabular}

Table III: Measurement results for the diversity metric $D$ at the three investigated frequencies and deviation $\Delta D(\%)$ of the values with regards to $\mathrm{D}$ for $3.1 \mathrm{GHz}$

that the performance of the system is reliable in the frequency band $[3.1 ; 10.6] \mathrm{GHz}$.

If the objective is to design a multiple antenna system for a wearable garment, we quickly run out of commonly used positions. The proposed method can be adjusted to the requirements of the application for positioning the antennas. For our case, we limited the available surface to the torso, but the method is still valid in case of adding or removing surfaces. Additionally, the method could be used for designing an antenna system in any frequency band.

The power imbalance between the separate links is a vital parameter for diversity systems and it should not exceed 10 $\mathrm{dB}$. We calculated this parameter for each antenna pair at all measurement frequencies. The minimum and maximum values in $\mathrm{dB}$ at each frequency are presented in Table IV. As we can see from the table, the values are lower than $10 \mathrm{~dB}$ for all cases.

Due to the similar and really small correlation values

\begin{tabular}{|c|c|c|c|c|c|c|c|c|c|}
\hline \multicolumn{10}{|c|}{ Power Imbalance, (dB) } \\
\hline \multicolumn{2}{|c|}{$3.1 \mathrm{GHz}$} & \multicolumn{2}{|c|}{$4 \mathbf{G H z}$} & \multicolumn{2}{|c|}{$5 \mathbf{G H z}$} & \multicolumn{2}{|c|}{$6 \mathbf{G H z}$} & \multicolumn{2}{|c|}{$6.85 \mathrm{GHz}$} \\
\hline $\min$ & $\max$ & $\min$ & $\max$ & $\min$ & $\max$ & $\min$ & $\max$ & $\min$ & $\max$ \\
\hline 0.06 & 8.31 & 0.49 & 9.29 & 0.35 & 9.30 & 0.06 & 9.12 & 0.07 & 9.53 \\
\hline \multicolumn{2}{|c|}{$7 \mathrm{GHz}$} & \multicolumn{2}{|c|}{$8 \mathbf{G H z}$} & \multicolumn{2}{|c|}{$9 \mathbf{G H z}$} & \multicolumn{2}{|c|}{$10 \mathrm{GHz}$} & \multicolumn{2}{|c|}{$10.6 \mathbf{G H z}$} \\
\hline $\min$ & $\max$ & $\min$ & $\max$ & $\min$ & $\max$ & $\min$ & $\max$ & $\min$ & $\max$ \\
\hline 0.08 & 8.94 & 0.12 & 6.45 & 0.04 & 6.66 & 0.11 & 4.95 & 0.02 & 4.28 \\
\hline
\end{tabular}

Table IV: Minimum and Maximum value of the power imbalance with regards to each of the measurement frequencies

$(\rho<0.18)$, the cumulative distribution functions (CDF) of the diversity combained signals for the three investigated frequencies are very similar to each other. Therefore, here we present the CDF only for $3.1 \mathrm{GHz}$ (Fig. 12). The difference in the diversity gain (Table $\mathrm{V}$ ) between a system with 5 antennas and one with 8 is only $1.4 \mathrm{~dB}$. Therefore, it is recommended to deploy no more than 5 antennas in a practical setup. From the same Table we can see that a system with 5 antennas, situated on the optimal antenna positions, can provide a diversity gain of more than $9.6 \mathrm{~dB}$. These values are calculated for systems with equally correlated branches, using [42]. As the branches in our system are not equally correlated, we consider as correlation value the highest $\rho$ between the nodes (Table V). The performance of the system with two antennas is comparable to previously published results on spatial diversity for UWB and BAN systems (e.g. [23], [43]), with the difference that we consider a simpler diversity combining technique. 
A benefit of this approach is the implementation of multiple

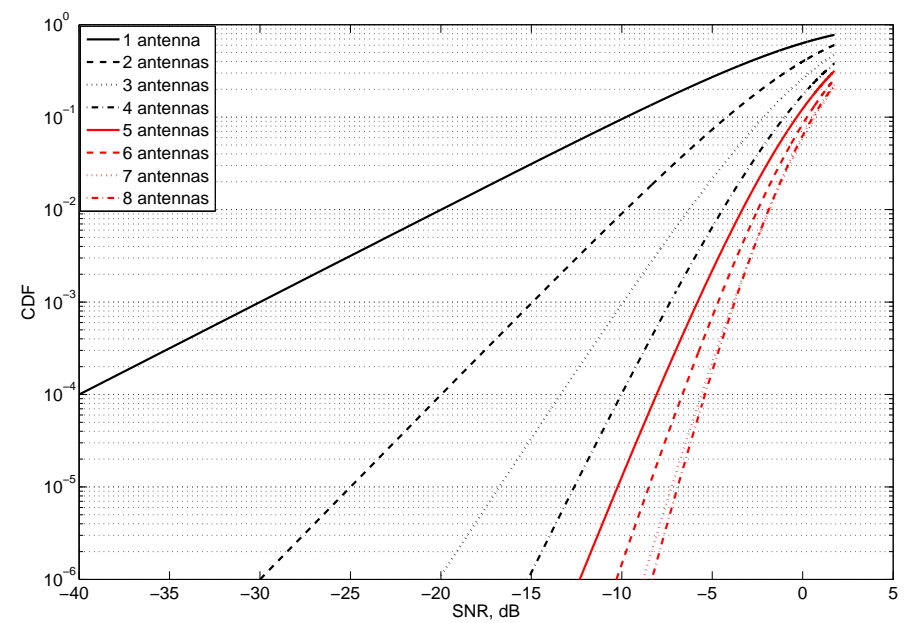

Figure 12: Cumulative Distribution Function of the diversity combined signals for $3.1 \mathrm{GHz}$

\begin{tabular}{|c||c|c||c|c||c|c|}
\hline \multicolumn{4}{|c|}{ Diversity gain (DG) for NLoS A1 In Building Environment for the three investigated frequencies } \\
\hline \multirow{2}{*}{$\begin{array}{c}\text { number of } \\
\text { antennas }\end{array}$} & \multicolumn{2}{|c|}{$3.1 \mathbf{~ G H z}$} & \multicolumn{2}{|c|}{$6.85 \mathbf{~ G H z}$} & \multicolumn{2}{|c|}{$10.6 \mathbf{G H z}$} \\
\cline { 2 - 7 } & $m a x \rho$ & $\mathbf{D G}[\mathbf{d B}]$ & $\max \rho$ & $\mathbf{D G}[\mathbf{d B}]$ & $\max \rho$ & $\mathbf{D G}[\mathbf{d B}]$ \\
\hline 2 antennas & 0.005 & 5.58 & 0.016 & 5.57 & 0.042 & 5.57 \\
\hline 3 antennas & 0.098 & 7.61 & 0.144 & 7.60 & 0.042 & 7.73 \\
\hline 4 antennas & 0.121 & 8.90 & 0.144 & 8.88 & 0.051 & 8.95 \\
\hline 5 antennas & 0.176 & 9.63 & 0.177 & 9.63 & 0.051 & 9.76 \\
\hline 6 antennas & 0.176 & 10.22 & 0.177 & 10.21 & 0.067 & 10.34 \\
\hline 7 antennas & 0.176 & 10.67 & 0.177 & 10.67 & 0.071 & 10.80 \\
\hline 8 antennas & 0.176 & 11.03 & 0.177 & 11.03 & 0.124 & 11.11 \\
\hline
\end{tabular}

Table V: Measurement results for the diversity gain at the three investigated frequencies

antennas (more than two) for spatial diversity for off-body BAN communications. Moreover, none of the cited contributions dealt with the important problem of how to optimally place the body-worn antennas, in order to maximize diversity performance. Therefore, the presented method aims to give an answer to such a problem. Further on, in contrast to most of the research on UWB BANs, which apply pulsed signals and focus on on-body communication, e.g. [15], [17], [18], [20], etc., our focus is on off-body MB-OFDM UWB systems. The use of MB-OFDM technique allows lower power consumption and longer propagation distances [7].

The limitations of the proposed method are the static nature of the simulations and the moderate walking speed of $1 \mathrm{~m} / \mathrm{s}$ of the test person during measurements. A mobile test subject that moves at higher speed will provide more diversity in the channel and will affect the characteristics of the system, such as antenna parameters, correlation, etc. Furthermore, at this point, the proposed approach investigates the behavior of the system for separate subcarriers. It will be beneficial to investigate how the system behaves throughout the whole bandwidth. Both aspects are considered for further research.

\section{CONCLUSIONS}

The use of UWB antennas and diversity techniques can improve the performance and the reliability of off-body BANs. In this paper, a method for determining the optimal positions of UWB nodes on the body is presented, providing a reliable diversity antenna system operating in the FCC band between 3.1 and $10.6 \mathrm{GHz}$. The placement of the nodes is determined by finding those positions that maximize the value of the diversity metric D.

Through simulations, we show that a system designed using the proposed method is reliable throughout the entire investigated bandwidth and that it can be used for both indoor and outdoor applications.

The measurement and simulated data for the optimal antenna positions agree very well, with the diversity metric deviating less than $9 \%$. For the optimal positions and the most commonly used positions, the largest deviation is found at 3.1 GHz. The method is beneficial for designing systems that operate in the lower part of the band. An advantage of the proposed method is its adjustability to the requirements of the application for positioning the antennas. The technique optimizes the position of the nodes in order to provide maximum diversity performance over the complete FCC band between $[3.1 ; 10.6] \mathrm{GHz}$, independent of the used wireless communication standard.

Using the proposed method, we recommend a system with five antennas, situated on the optimal antenna positions. Such a system can provide a diversity gain of more than $9.6 \mathrm{~dB}$. Further research will consist of extending the measurement data by including channel sounding experiments and investigating the influence of the antenna by comparing the performance of the system when we use different antenna topologies.

\section{REFERENCES}

[1] R. Verdone and A. Zanella, Eds., Pervasive Mobile and Ambient Wireless Communications. Springer, 2012.

[2] C. Oliveira and L. M. Correia, "Perspectives for the use of MIMO in Dynamic Body Area Networks," in Antennas and Propagation (EuCAP), 2013 7th European Conference on, April 2013, pp. 482-486.

[3] R. D’Errico and L. Ouvry, "A Statistical Model for On-body Dynamic Channels," Intenational Journal of Wireless Information Networks, vol. 17, pp. 99-104, December 2010.

[4] M. Kim and J. Takada, "Statistical Model for 4.5-GHz Narrowband OnBody Propagation Channel With Specific Actions," IEEE Antennas and Wireless Propagation Letters, vol. 8, pp. 1250-1254, 2009.

[5] L. C. Tran and A. Mertins, "Space-Time-Frequency Code Implementations in MB-OFDM UWB Communications: Design Criteria and Performance," IEEE Transactions on Wireless Communications, vol. 8, no. 2, pp. 701-713, February 2009.

[6] W. P. Siriwongpairat, W. Su, M. Olfat, and K. J. R. Liu, "MultibandOFDM MIMO Coding Framework for UWB Communication Systems," IEEE Transactions on Signal Processing, vol. 54, no. 1, pp. 214-224, January 2006.

[7] W. P. Siriwongpairat and K. J. R. Liu, Ultra-wideband communication systems: multiband OFDM approach. Hoboken, New Jersey: John Wiley \& Sons, Inc.-IEEE Press, December 2007.

[8] S. L. Cotton and W. G. Scanlon, "Channel Characterization for Singleand Multiple-Antenna Wearable Systems Used for Indoor Body-to-Body Communications," IEEE Transactions on Antennas and Propagation, vol. 57, no. 4, pp. 980-990, April 2009.

[9] R. Rosini and R. D’Errico, “Comparing On-Body dynamic channels for two antenna designs," in Antennas and Propagation Conference (LAPC), 2012 Loughborough, November 2012, pp. 1-4.

[10] E. Reusens, W. Joseph, B. Latré, B. Braem, G. Vermeeren, E. Tanghe, L. Martens, I. Moerman, and C. Blondia, "Characterization of OnBody Communication Channel and Energy Efficient Topology Design for Wireless Body Area Networks,' IEEE Transactions on Information Technology in Biomedicine, vol. 13, no. 6, pp. 933-945, November 2009. 
[11] L. Liu, R. D’Errico, L. Ouvry, P. De Doncker, and C. Oestges, "Dynamic Channel Modeling at $2.4 \mathrm{GHz}$ for On-Body Area Networks," Advances in Electronics and Telecommunications, vol. 2, no. 4, pp. 18-27, December 2011.

[12] M. Mackowiak and L. M. Correia, "Correlation analysis of off-body radio channels in a street environment," Personal Indoor and Mobile Radio Communications (PIMRC), 2012 IEEE 23rd International Symposium on, pp. 1769-1773, September 2012.

[13] A. Alomainy, Y. Hao, A. Owadally, C. G. Parini, Y. Nechayev, C. C. Constantinou, and P. S. Hall, "Statistical Analysis and Performance Evaluation for On-Body Radio Propagation With Microstrip Patch Antennas," Antennas and Propagation, IEEE Transactions on, vol. 55, no. 1, pp. 245-248, January 2007.

[14] S. van Roy, F. Quitin, L. Liu, C. Oestges, F. Horlin, J. Dricot, and P. De Doncher, "Dynamic Channel Modeling for Multi-Sensor Body Area Networks," Antennas and Propagation, IEEE Transactions on, vol. 61, no. 4, pp. 2200-2208, April 2013.

[15] S. van Roy, C. Oestges, F. Horlin, and P. D. Doncker, "A Comprehensive Channel Model for UWB Multisensor Multiantenna Body Area Networks," IEEE Transactions on Antennas and Propagation, vol. 58, no. 1, pp. 163-170, January 2010.

[16] Y. Chen, J. Teo, J. C. Y. Lai, E. Gunawan, K. S. Low, C. B. Soh, and P. B. Rapajic, "Cooperative Communications in Ultra-Wideband Wireless Body Area Networks: Channel Modeling and System Diversity Analysis," IEEE Journal on Selected Areas in Communications, vol. 27, no. 1, pp. 5-16, January 2009.

[17] S. van Roy, C. Oestges, F. Horlin, and P. D. Doncker, "On-body Propagation Velocity Estimation Using UWB Frequency Domain Spatial Correlation Analyses," Electronics Letters, vol. 43, no. 25, pp. 14051406, December 2007.

[18] Q. H. Abbasi, M. M. Khan, S. Liaqat, M. Kamran, A. Alomainy, and Y. Hao, "Experimental Investigation of Ultra Wideband Diversity Techniques for Antennas and Radio Propagation in Body-Centric Wireless Communications," Progress in Electromagnetics Research C, vol. 34, pp. 165-181, January 2013.

[19] Q. H. Abbasi, A. Alomainy, and Y. Hao, "Ultra Wideband Antenna Diversity Techniques for On/Off-Body Radio Channel Characterisation," IEEE International Workshop on Antenna Technology, pp. 209-212, March 2012

[20] W.-J. Chang, J.-H. Tarng, and S.-Y. Peng, "Frequency-SpacePolarization on UWB MIMO Performance for Body Area Network Applications," IEEE Antennas and Wireless Propagation Letters, vol. 7, pp. 577-580, May 2008.

[21] Q. H. Abbasi, A. Alomainy, and Y. Hao, "Antenna Diversity Technique for Enhanced Ultra Wideband Radio Performance in Body-Centric Wireless Networks," Wireless Technology Conference (EuWIT), 2010 European, pp. 197-200, September 2010.

[22] I. Khan and P. S. Hall, "Multiple Antenna Reception at 5.8 and 10 $\mathrm{GHz}$ for Body-Centric Wireless Communication Channels," Antennas and Propagation, IEEE Transactions on, vol. 57, no. 1, pp. 248-255, January 2009.

[23] A. J. Ali, S. L. Cotton, and W. G. Scanlon, "Spatial Diversity for Off-Body Communications in an Indoor Populated Environment at 5.8 GHz," in Antennas \& Propagation Conference, 2009. LAPC 2009. Loughborough, November 2009, pp. 641-644.

[24] M. M. Khan, Q. H. Abbasi, A. Alomainy, and Y. Hao, "Study of Line of Sight (LOS) and None Line of Sight (NLOS) Ultra Wideband OffBody Radio Propagation for Body Centric Wireless Communications in Indoor,' in Antennas and Propagation (EUCAP), Proceedings of the 5th European Conference on, April 2011, pp. 110-114.

[25] A. A. Goulianos, T. W. C. Brown, B. G. Evans, and S. Stavrou, "Wideband Power Modeling and Time Dispersion Analysis for UWB Indoor Off-Body Communications," Antennas and Propagation, IEEE Transactions on, vol. 57, no. 7, pp. 2162-2171, July 2009.

[26] New Public Safety Applications and Broadband Internet Access among Uses Envisioned by FCC Authorization of Ultra-Wideband Technology, Federal Communications Commission, February 2002, www.fcc.gov.

[27] A. F. Molisch, D. Cassioli, C.-C. Chong, S. Emami, A. Fort, B. Kannan, J. Karedal, J. Kunisch, H. G. Schantz, K. Siwiak, and M. Z. Win, "A Comprehensive Standardized Model for Ultrawideband Propagation Channels," IEEE Transactions on Antennas and Propagation, vol. 54, no. 11, pp. 3151-3166, November 2006.

[28] R. T. Al-Zubi and M. Krunz, "Interference Management and Rate Adaptation in OFDM-Based UWB Networks," IEEE Transactions on Mobile Computing, vol. 9, no. 9, pp. 1267-1279, September 2010.

[29] L. Hentillä, P. Kyösti, M. Käske, M. Narandzic, and M. Alatossava, MATLAB implementation of the WINNER Phase
II Channel Model verl.1 , December 2007, https://www.istwinner.org/phase 2 model.html.

[30] G. Vermeeren, W. Joseph, and L. Martens, "Statistical Multi-path Exposure Model for Assessing the Whole-body SAR in a Heterogeneous Human Body Model in a Realistic Environment," Bioelectromagnetics, vol. 34, no. 3, pp. 240-251, April 2013.

[31] SEMCAD X, Speag Schmid \& Partner Engineering AG, 2012 , http://www.semcad.com.

[32] A. Thielens, H. D. Clercq, S. Agneessens, J. Lecoutere, L. Verloock, F. Declercq, G. Vermeeren, Emmeric, H. Rogier, R. Puers, L. Martens, and W. Joseph, "Personal Distributed Exposimeter for Radio Frequency Exposure Assessment in Real Environments." Bioelectromagnetics, vol. 34, no. 7, pp. 563-567, October 2013.

[33] W. Dullaert and H. Rogier, "Novel Compact Model for the Radiation Pattern of UWB Antennas Using Vector Spherical and Slepian Decomposition," Antennas and Propagation, IEEE Transactions on, vol. 58, no. 2, pp. 287-299, February 2010.

[34] G. Vermeeren, W. Joseph, C. Olivier, and L. Martens, "Statistical Multipath Exposure of a Human in a Realistic Electromagnetic Environments," Health Physics, vol. 94, no. 4, pp. 345-354, April 2008.

[35] A. Thielens, G. Vermeeren, W. Joseph, and L. Martens, "Stochastic Method for Determination of the Organ-specific Averaged SAR in Realistic Environments at $950 \mathrm{MHz}$,"Bioelectromagnetics, vol. 34, no. 7, pp. 549-562, October 2013.

[36] R. G. Vaugham and J. B. Andersen, "Antenna Diversity in Mobile Communications," Vehicular Technology, IEEE Transactions on, vol. 36, no. 4, pp. 149-172, November 1987.

[37] R. Vaugham and J. B. Andersen, Channels, Propagation and Antennas for Mobile Communications, first edition ed., ser. IET Electromagnetic Waves Series, P. P. Clarricoats and P. E. Jull, Eds. London, Unated Kingdom: The Institution of Engineering and Technology, 2006, vol. 50.

[38] C. Oliveira and L. M. Correia, "Signal Correlation and Power Imbalance in Dynamic On-Body Communications," in Vehicular Technology Conference (VTC Spring), 2013 IEEE 77th, June 2013, pp. 1-5.

[39] M. T. Ivrlač and J. A. Nossek, "Quantifying Diversity and Correlation in Rayleigh Fading MIMO Communication Systems," in Signal Processing and Information Technology, 2003. ISSPIT 2003. Proceedings of the 3rd IEEE International Symposium on, December 2003, pp. 158-161.

[40] A. Christ, W. Kainz, E. G. Hahn, K. Honegger, M. Zefferer, E. Neufeld, W. Rascher, R. Janka, W. Bautz, J. Chen, B. Kiefer, P. Schmitt, H.-P. Hollenbach, J. Shen, M. Oberle, D. Szczerba, A. Kam, J. W. Guag, and N. Kuster, "The Virtual Family - development of surface based anatomical models of two adults and two children for dosimetric simulations," Physics in Medicine and Biology, vol. 55, no. 2, pp. N23N38, January 2010.

[41] N5242A PNA-X Microwave Network Analyzer, Agilent Technologies, http://www.home.agilent.com/en/pd-867173-pn-N5242A/pna-Xmicrowave-network-analyzer?nid=-536902643.426031\&cc $=\mathrm{BE} \& \mathrm{lc}=\mathrm{dut}$

[42] Y. Chen and C. Tellambura, "Distribution Functions of Selection Combiner Output in Equally Correlated Rayleigh, Rician, and Nacagami$m$ Fading Channels," Communications, IEEE Transactions on, vol. 52, no. 11, pp. 1948-1956, November 2004.

[43] A. Sibille and S. Bories, "Spatial Diversity for UWB Communications," 5th European Personal Mobile Communications Conference, pp. 367370, April 2003. 